\title{
Mercury in pediatric poisoning
}

\author{
Gianpaolo Guzzi • Chiara Marsili • Paolo D. Pigatto
}

Received: 24 December 2009 / Accepted: 11 February 2010/Published online: 1 March 2010

(C) Springer-Verlag 2010

\section{Dear Editor,}

The interesting short report of Erkek et al. [1] describes a 10 -year-old girl with inorganic mercury intoxication, possibly because of inorganic mercury overexposure while attempting to swim into a creek of cultivated area. Perhaps the reason for very high levels of mercury in the creek waters was due to improper mercury disposal.

In the Discussion section, the authors state that "...... findings of hemolysis disappeared rapidly which was consisted with inorganic mercury intoxication" [1]. It has been reported that both inorganic mercury (mercuric mercury, $\mathrm{Hg}^{2+}$ ) and organic mercury (methyl mercury, $\mathrm{CH}_{3} \mathrm{Hg}^{+}$) are able to induce rapid hemolysis in human erythrocyte in vitro [2]. It is difficult to attribute causation of hemolysis to inorganic mercury alone.

With regard to the chemical identification of mercury involved in this case of poisoning, in the absence of mercury speciation, which would have allowed to know the exact chemical nature of mercury, the assessment of mercury red blood cell (RBC)/plasma distribution analysis would have provided more useful data.

G. Guzzi $(\bowtie) \cdot$ P. D. Pigatto

Italian Association for Metals and Biocompatibility Research, A.I.R.M.E.B,

Via A. Banfi, 4,

20122 Milan, Italy

e-mail: gianpaolo_guzzi@fastwebnet.it

C. Marsili • P. D. Pigatto

Department of Technology for Health, Dermatological Clinic, IRCCS Galeazzi Hospital, University of Milan,

Milan, Italy
It is well known that a ratio of 1:1 may suggest a high probability of exposure to inorganic mercury. By contrast, if the mercury is present mainly in the RBC compartment (by a plasma to $\mathrm{RBC}$ ratio of 1:10), it is highly suggestive of previous overexposure to organic mercury. This is a simple solution to a problem in clinical toxicology.

Authors suspected inorganic mercury poisoning, and if it is so, why, therefore, was the child subjected to a chelation therapy with DMSA instead of DMPS? DMPS is more specific for inorganic mercury [3]. A case of hemolytic anemia has been reported associated with the use of DMSA in a patient with G6PD deficiency. Blood test for G6PD may be useful before using DMSA [3].

This valuable case report highlights the complexity of diagnosis and treatment of serious mercury poisoning.

Conflict of interest statement We have no conflicts of interest connected with this work. The authors declare that they have no competing financial interest.

Funding None.

\section{References}

1. Erkek N, Senel S, Sarac A et al (2009) Being alive after a severe inorganic mercury intoxication. Eur J Pediatr. doi:10.1007/s00431009-1073-2

2. Ichikawa H, Ronowicz K, Hicks M, Gebicki JM (1987) Lipid peroxidation is not the cause of lysis of human erythrocytes exposed to inorganic or methylmercury. Arch Biochem Biophys 259:46-51

3. Guzzi G, La Porta CA (2008) Molecular mechanisms triggered by mercury. Toxicology 244:1-12 